\title{
ERRORS IN THE VICKERS DIAGONAL LENGTH MEASUREMENT CAUSED BY DIFFERENT DESIGNS OF MICROSCOPES
}

\author{
S. Takagi ${ }^{1}$, Y. Tanaka ${ }^{2}$, Y. Seino ${ }^{3}$ \\ ${ }^{1}$ National metrology institute of Japan, AIST, Tsukuba, Japan, satoshi.takagi@aist.go.jp \\ ${ }^{2}$ National metrology institute of Japan, AIST, Tsukuba, Japan, yukimi.tanaka@ aist.go.jp \\ ${ }^{3}$ National metrology institute of Japan, AIST, Tsukuba, Japan, y.seino@aist.go.jp
}

\begin{abstract}
:
The errors in the Vickers diagonal length measurement are discussed from the point of view of the design of microscope. Effects of the numerical aperture (NA) of an objective lens are evaluated through the experiment. With smaller NA, the resolving power of the microscope gets worse and the depth of focus gets deeper as predicted Rayleigh's criterion. The relationship between the repeatability of diagonal length and the objective NA follows the theory. In addition, the variation of NA relates the measured diagonal length. Those effects are much significant than the image resolution or the minimum count of a measuring system.
\end{abstract}

Keywords: Vickers hardness; diagonal length; diffraction limits; resolution; uncertainty of measurement

\section{INTRODUCTION}

Consider establishing a national primary Vickers hardness standard where any reference materials are not available, the error in diagonal length measurement is one of the most significant sources of measurement uncertainty as well as testing force, testing cycle, uniformity of reference blocks or repeatability of measurement. Although the ISO standard [1] requires to consider "both the resolution of the length measurement indicating instrument and the optical resolution of the measuring microscope", most of discussions are focused on the indicating instrument $(e . g$., the discussion in the EA committee) and quite few studies concerned the resolving power of the microscope [2]. In reality, the same size of indentation is measured with different magnification or different numerical aperture depending to the design of the measuring device each calibration laboratory used. In this paper, possible errors caused by the design of an optical microscope are discussed.

\section{MICROSCOPE DESIGN FACTORES AND THEIR EFFECTS ON MEASUREMENT}

Typical design factors of a microscope are the objective magnification and its numerical aperture. Usually, those are given as specifications of an objective lens and users cannot change those numbers by themselves. Here, the effects of those design factors are briefly reviewed.

\subsection{Magnification}

The magnification directly affects to the resolution of readings whichever it is detected by a camera or a human eye.

\subsection{Numerical aperture}

The numerical aperture (NA) is the index related to the half-angle of the cone of the light that can enter the objective

$\mathrm{NA}=n \times \sin$

where $n$ is the index of refraction ( $n=1$ in air). It determines the resolving power , i. e., diffraction limit, by following equation [3]

$$
=0.61 \times \frac{}{\mathrm{NA}} \text {, }
$$

where is the wavelength of light. When the visible light is considered, $=550 \mathrm{nmis}$ often used. The NA also affects the depth of focus (DOF) of a microscope by following equation.

$$
\mathrm{DOF}=\frac{n}{2 \times \mathrm{NA}^{2}}
$$

When a microscope makes focus on a sample, the distance between the objective and the sample can be varied within DOF. From a simple geometrical consideration, an image will expand by the difference of the distance and the maximum value is estimated as follows.

$$
=\mathrm{DOF} \times \mathrm{an} \text {. }
$$

\section{EXPERIMENT}

In order to investigate the effects of microscope design factors, a series of measurement of Vickers 
indentations was carried out. Photos of indentations on a hardness reference blocks were taken by an Olympus BXFM optical microscope. In order to discuss effects of magnification and NA separately, single objective lens of $50 \times$ magnification was used. This microscope system is infinity-corrected, $i$. e., the beam path is parallel between the objective and tube lenses (Figure 1). By setting a diaphragm into this parallel beam section, its pupil diameter, therefore its NA, can be changed without changing the objective magnification.

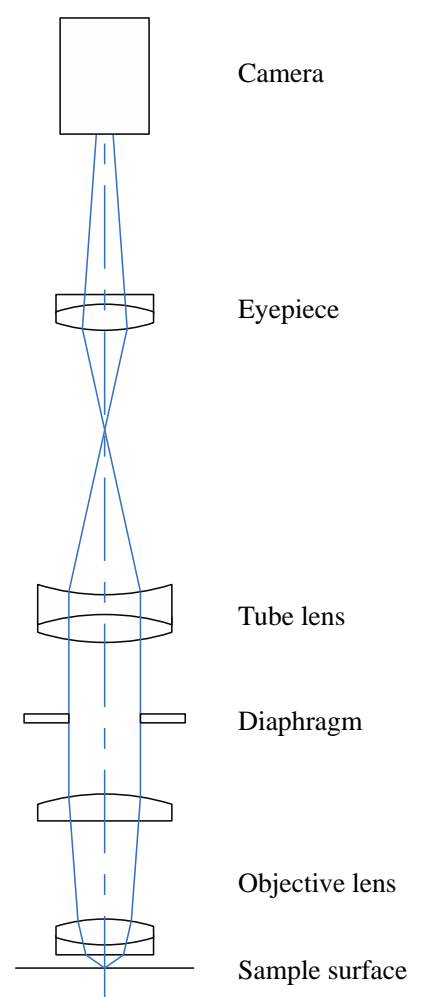

Figure 1: Infinity-corrected microscope with a replace able diaphragm

Table 1: Experimental conditions

\begin{tabular}{lllll}
\hline $\begin{array}{l}\text { Pupil } \\
\text { diameter, } \\
\mathbf{m m}\end{array}$ & NA & $\begin{array}{l}\text { Resolving } \\
\text { power } \boldsymbol{\delta}, \\
\boldsymbol{\mu m}\end{array}$ & DOF, $\boldsymbol{\mu m}$ & $\begin{array}{l}\text { Maxium } \\
\text { expansion } \\
\boldsymbol{\varepsilon}, \boldsymbol{\mu m}\end{array}$ \\
\hline 3.6 & 0.50 & 0.7 & 0.4 & 0.6 \\
3.0 & 0.42 & 0.8 & 0.5 & 0.7 \\
2.5 & 0.35 & 1.0 & 0.6 & 0.8 \\
1.9 & 0.26 & 1.3 & 0.8 & 1.1 \\
1.3 & 0.18 & 1.9 & 1.1 & 1.6
\end{tabular}

Images were taken in a format of $2592 \times 1944$ pixels and diagonal lengths of indentation were measured on a computer screen. The size of image was calibrated by taking an image of calibrated stage micrometer. The pixel size is $0.096 \mu \mathrm{m}$.

In this experiment, indentation of $200 \mathrm{HV} \mathrm{1,600}$ HV 10 and $900 \mathrm{HV} 10$ on hardness reference blocks were chosen. The typical diagonal lengths of indentations are $96 \mu \mathrm{m}, 176 \mu \mathrm{m}$ and $144 \mu \mathrm{m}$, respectively, which fit the field of view with the same $50 \times$ objective lens. Those indentations were observed in five different conditions of optical system shown in Table 1. To evaluate the repeatability of focusing, a displacement transducer, Mitutoyo LGH-110, with $0.01 \mu \mathrm{m}$ resolution was attached on the microscope column and the position of the microscope tube was recorded corresponding to every shot of image.

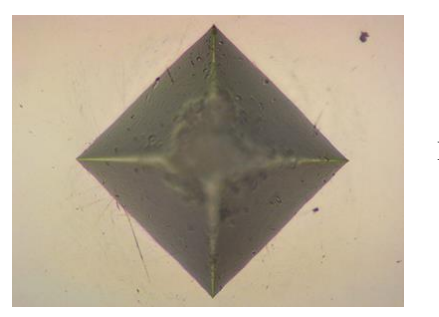

$\mathrm{NA}=0.50$

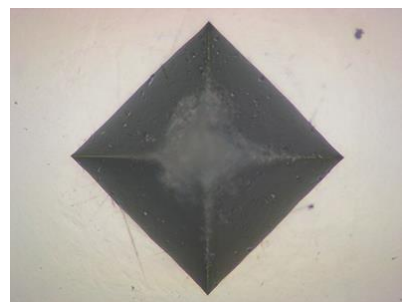

$\mathrm{NA}=0.42$

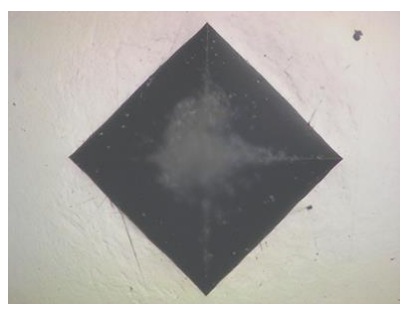

$\mathrm{NA}=0.35$

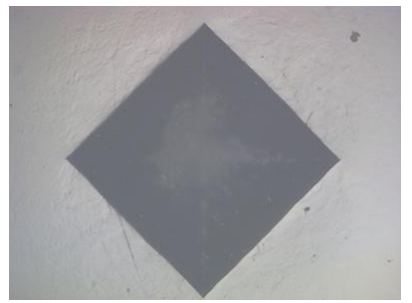

$\mathrm{NA}=0.26$

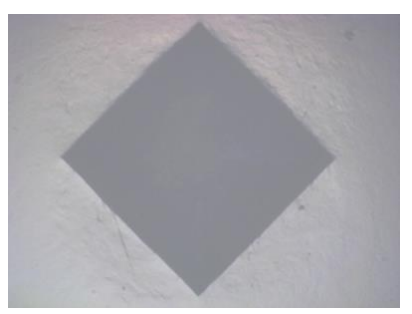

$\mathrm{NA}=0.18$

Figure 2: Images of an indentation taken in dif ferent NA setting (600 HV 10).

\section{RESULTS}

Figure 2 shows the difference of visibility of observed indentation with different NA setting. With larger NA, the image contrast is higher and the details of sample's geometrical features can be seen clearer. On the other hand, with smaller NA, the contrast is lower because the light intensity collected by the microscope is weaker. In addition, the inside of the indentation is blacked out and only the perimeter of indentation can be observed. The 
most important fact is that the image got blurred when smaller NA is chosen. It makes difficult to determine the exact position of the indentation corner and increases the uncertainty of diagonal length measurement.

Table 2: ANOVA of the diagonal length measurement for $200 \mathrm{HV} 1$ indentation

\begin{tabular}{lllll}
\hline Source & SS & df & MS & $\mathbf{F}_{\mathbf{0}}$ \\
\hline NA & 2.795 & 4 & 0.70 & $6.2^{* *}$ \\
Orientation & 0.115 & 1 & 0.11 & 1.0 \\
Error & 4.996 & 44 & 0.11 & \\
\hline Total & 7.905 & 49 & & \\
\hline
\end{tabular}

** Significant at $99 \%$ confidence level

Table 3: ANOVA of the diagonal length measurement for $600 \mathrm{HV} 10$ indentation

\begin{tabular}{lllll}
\hline Source & SS & df & MS & $\mathbf{F}_{\mathbf{0}}$ \\
\hline NA & 3.802 & 4 & 0.95 & $15.7^{* *}$ \\
Orientation & 1.874 & 1 & 1.87 & $30.9^{* *}$ \\
Error & 2.670 & 44 & 0.06 & \\
\hline Total & 8.347 & 49 & & \\
\hline
\end{tabular}

** Significant at $99 \%$ confidence level

Table 4: ANOVA of the diagonal length measurement for $900 \mathrm{HV} 10$ indentation

\begin{tabular}{lllll}
\hline Source & SS & df & MS & $\mathbf{F}_{\mathbf{0}}$ \\
\hline NA & 1.465 & 4 & 0.37 & $8.0^{* *}$ \\
Orientation & 0.294 & 1 & 0.29 & $6.4^{*}$ \\
Error & 2.014 & 44 & 0.05 & \\
\hline Total & 3.773 & 49 & & \\
\hline
\end{tabular}

* Significant at $95 \%$ confidence level

** Significant at $99 \%$ confidence level

Two diagonals were measured for an indentation, $i$. e., longitudinal length, $d_{1}$ and lateral length, $d_{2}$. Conceptually, those two diagonal lengths should be same. However, in this experiment (and maybe in the most of other practical cases) they are not always identical. To eliminate the effect of measuring orientation and evaluate the uncertainty of measurement associated with the optical system only, whole set of data were analyzed by two-factor analysis of variance (ANOVA) with replication. The results were shown in Tables 2 to 4 . They show that the effect of NA is highly significant in all indentations. At the same time the effect of measuring orientation cannot be ignored for $600 \mathrm{HV}$ 10 and $900 \mathrm{HV} 10$ indentations. Therefore, following analysis are based on the measurement variation excluding the effect of measuring orientation. The standard deviation of error is $0.34 \mu \mathrm{m}, 0.25 \mu \mathrm{m}$ and $0.21 \mu \mathrm{m}$ for $200 \mathrm{HV} 1,600$ HV 10 and $900 \mathrm{HV} 10$ indentations, respectively.

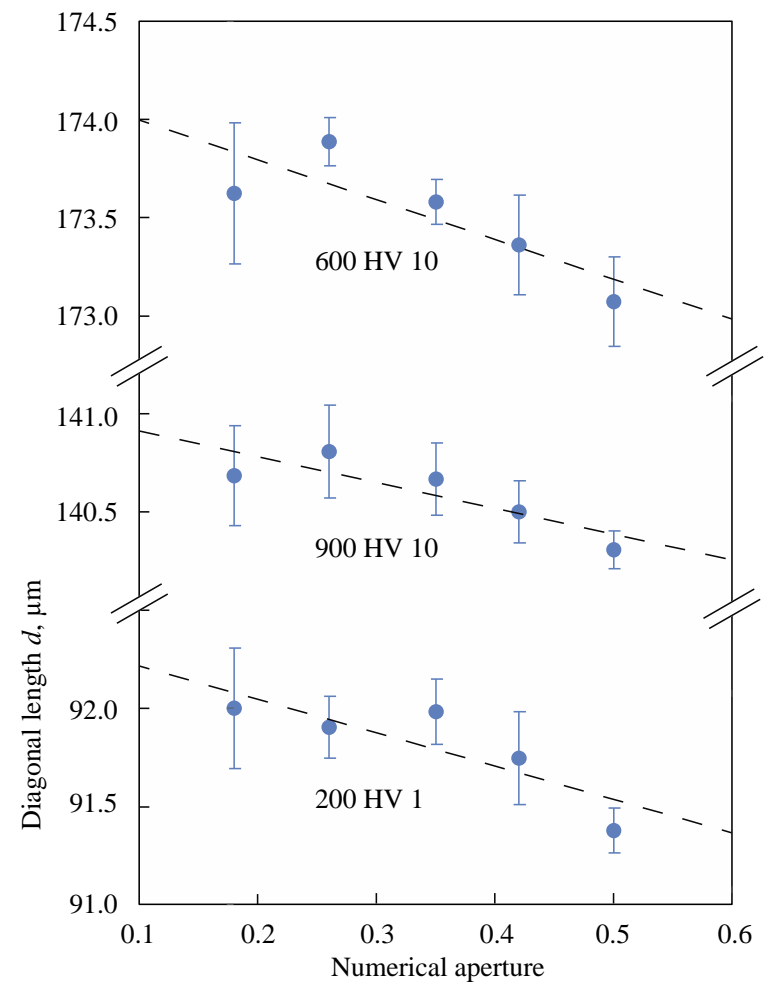

Figure 3: Diagonal length measured with different numerical aperture

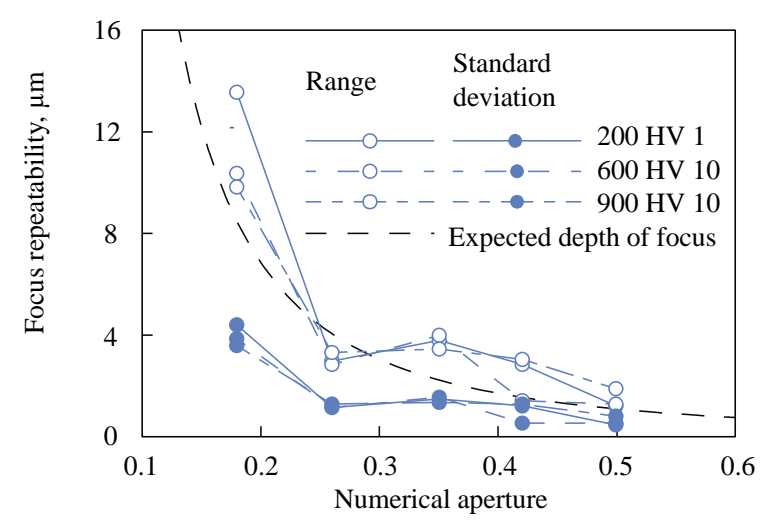

Figure 4: Repeatability of the microscope focussing

Measured average diagonal lengths in different NA are shown in Figure 3. It indicates the diagonal length get longer as NA decreases and this same trend is confirmed for all three indentations. It is not easy to explain the relationship between measured diagonal length and NA, it seems that there is an effect of blurred images to overestimate the diagonal length. Even though a theoretical model of this error has not been established, when a linear equation is fitted for the data, the sensitivity will be -0.13 to $-0.20 \mu \mathrm{m}$ per 0.1 unit of NA.

The repeatability of focusing is shown in Figure 4. There is a clear trend that the repeatability gets better as NA increases. It indicates that the position of the microscope tube distributed in the range almost same with expected depth of focus of the microscope. 


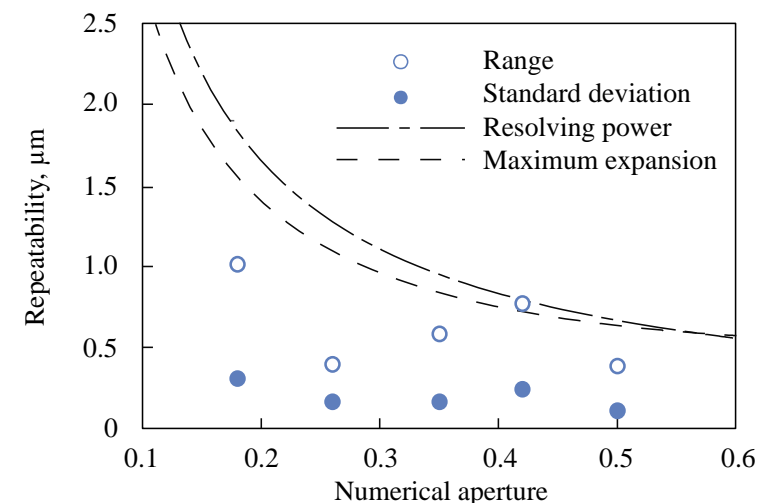

(a) $200 \mathrm{HV} 1$

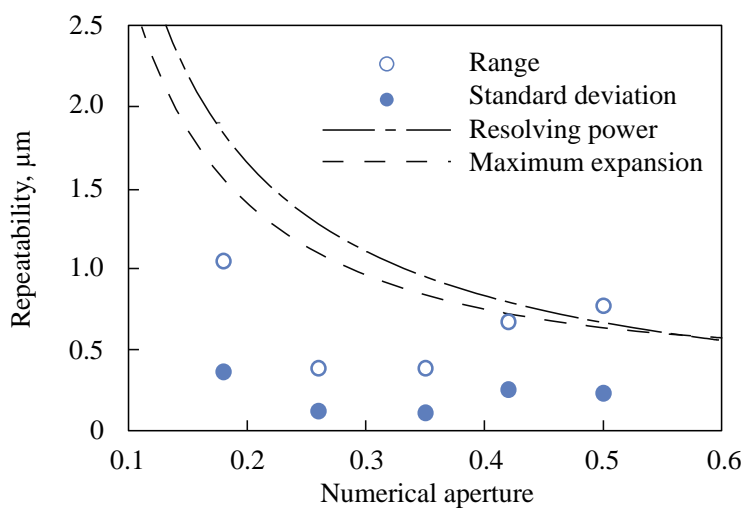

(b) $600 \mathrm{HV} 10$

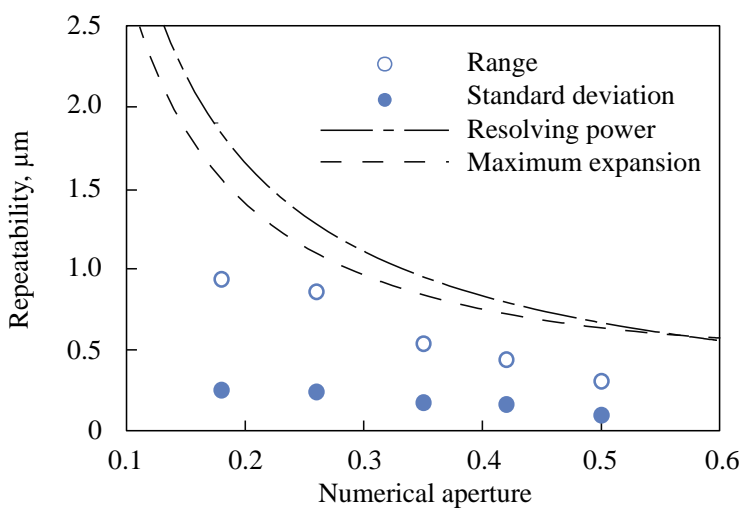

(c) $900 \mathrm{HV} 10$

Figure 5: Repeatability of diagonal length measurement in different NAs

As described in the previous section, the variation of the distance between the sample and the objective lens causes the expansion of the image and it can affect the diagonal length measurement. Figure 5 shows the repeatability of diagonal length readings (standard deviation and range) as well as the resolving power of microscope and the maximum expansion of image. The effects of the resolving power and the image expansion due to the uncertain focusing are similar and cannot be separated in this experiment. However, the variation of the diagonal length measurement seems to distribute within those two criteria.

\section{DISCUSSIONS}

The ISO 6507-2 describes the uncertainty estimation of the calibration results of hardness testing system. In the calibration of the diagonal measuring system, it requires to include the uncertainty due to the resolution of the measuring system. However, it is not clear what the resolution exactly means. In the experiment in this paper, the image resolution is $0.096 \mu \mathrm{m}$. The primary standard Vickers hardness machine in our laboratory, Mitutoyo SHT-41, has a digital counter on its measuring system and it can detect the minimum of $0.006 \mu \mathrm{m}$ of length with $40 \times$ objective. The results shown in this paper suggest the measurand distributes within the range of the resolving power of microscope which can be predicted through Rayleigh's criterion. As a result, the repeatability of measurement was $0.21 \mu \mathrm{m}$ to $0.34 \mu \mathrm{m}$. It is much larger than the image resolution or the minimum count of the instrument. In addition, the result shows the diagonal length itself can vary with NA of the objective lens. The NA of microscope objective is typically ranging 0.2 to 0.9 , according to its magnification and working distance. Our result suggests the evaluated diagonal length can vary in the range of $0.9 \mu \mathrm{m}$ to $1.4 \mu \mathrm{m}$, which is the amount of bias we cannot ignore. Further consideration is expected in the guideline to estimate the uncertainty of measurement of a Vickers hardness testing machine.

\section{CONCLUTIONS}

From a series of experiments of the diagonal length measurement of Vickers indentation, the authors had following findings.

The NA of objective should have larger as possible. In general, it requires larger magnification. Such objective brings higher resolving power and narrower DOF. Both are contributing to reduce the repeatability of readings. Lower resolving power makes an image blurred and expanded, so that the measured diagonal length will be larger.

The discussion in the paper is about the resolving power of optical system but not the image resolution and the former is much larger than the latter. It is recommended to consider the resolving power of the optical system is included in every uncertainty budget of Vickers hardness standard.

\section{REFERENCES}

[1] ISO 6507-2: 2018.

[2] L. Brice, F. Davis, A. Crawshaw, NPL Report CMAM 87 (2003)

[3] L. Levi, Applied Optics, A Guide to Optical System Design, Vol. 1, John Wiley \& Sons (1968) New York. 